Article

\title{
Application of Fixed-Point Theory for a Nonlinear Fractional Three-Point Boundary-Value Problem
}

\author{
Ehsan Pourhadi ${ }^{1,2}$, Reza Saadati ${ }^{2}(1)$ and Sotiris K. Ntouyas $3,4, *$ (D) \\ 1 International Center for Mathematical Modelling in Physics and Cognitive Sciences, Department of \\ Mathematics, Linnaeus University, SE-351 95 Växjö, Sweden; epourhadi@alumni.iust.ir \\ 2 Department of Mathematics, Iran University of Science and Technology, Narmak, Tehran 16846-13114, Iran; \\ rsaadati@eml.cc \\ 3 Department of Mathematics, University of Ioannina, 45110 Ioannina, Greece \\ 4 Nonlinear Analysis and Applied Mathematics (NAAM)-Research Group, Department of Mathematics, \\ Faculty of Science, King Abdulaziz University, P.O. Box 80203, Jeddah 21589, Saudi Arabia \\ * Correspondence: sntouyas@uoi.gr
}

Received: 10 February 2019; Accepted: 6 June 2019; Published: 10 June 2019

Abstract: Throughout this paper, via the Schauder fixed-point theorem, a generalization of Krasnoselskii's fixed-point theorem in a cone, as well as some inequalities relevant to Green's function, we study the existence of positive solutions of a nonlinear, fractional three-point boundary-value problem with a term of the first order derivative

$$
\begin{gathered}
\left({ }_{a}^{C} D^{\alpha} x\right)(t)=f\left(t, x(t), x^{\prime}(t)\right), a<t<b, \quad 1<\alpha<2, \\
x(a)=0, x(b)=\mu x(\eta), a<\eta<b, \quad \mu>\lambda,
\end{gathered}
$$

where $\lambda=\frac{b-a}{\eta-a}$ and ${ }_{a}^{C} D^{\alpha}$ denotes the Caputo's fractional derivative, and $f:[a, b] \times \mathbb{R} \times \mathbb{R} \rightarrow \mathbb{R}$ is a continuous function satisfying the certain conditions.

Keywords: three-point boundary-value problem; Caputo's fractional derivative; Riemann-Liouville fractional integral; fixed-point theorems

\section{Introduction}

In the last decade, questions on positive solutions to two-point, three-point, and multi-point boundary value problems (BVPs) and integral boundary-value problems for nonlinear ordinary and fractional differential equations have attracted much interest. The investigation of three-point BVPs for nonlinear integer-order ordinary differential equations was initially begun by Gupta [1]. Since then, several authors have put their focus on the existence and multiplicity of solutions (or positive solutions) of three-point BVPs for nonlinear integer-order ordinary differential equations. Several papers are available in regard to the setting of integer orders of differential equations in the literature. In 2000, applying the fixed-point index theorems, the Leray-Schauder degree, and upper and lower solutions, Ma [2] studied a class of second-order three-point boundary value problems with a nonlinear term $f(x)$. In 2002, He and Ge [3], with the help of the Leggett-Williams fixed-point theorem [4], investigated the multiplicity of positive solutions of a problem with the nonlinear term $f(t, x)$ (see [5-15] and the references therein).

In recent years, multi-point boundary value problems have also been considered for fractional-order differential equations. For instance, employing the superlinearity and sublinearity, together with the well-known Guo-Lakshmikantham fixed-point theorem in cones, Ntouyas and 
Pourhadi [16] studied the existence of positive solutions to the boundary-value problem with a fractional order, $1<\alpha<2$. Furthermore, they investigated the convexity and concavity of the solutions with respect to the behavior of a given function as a coefficient of the subjected problem (see also [17-21]).

There were only a few papers available which focused on the existence of solutions for nonlinear fractional differential equations associated with three-point boundary conditions, which served as motivation for this work. The key idea of the current paper is that a term of the first-order derivative is involved in the subjected nonlinear problem, while most works (either fractional or ordinary differential equations) are done under the assumption that the first-order derivative is not involved explicitly in the nonlinear term.

In this paper, an analogy with a boundary-value problem for differential equations of integer orders via the Schauder fixed-point theorem, a generalized version of Krasnoselskii's fixed-point theorem in a cone [22], and also using the associated Green's function for the relevant problem, the existence of positive solutions for a fractional three-point boundary-value problem is investigated.

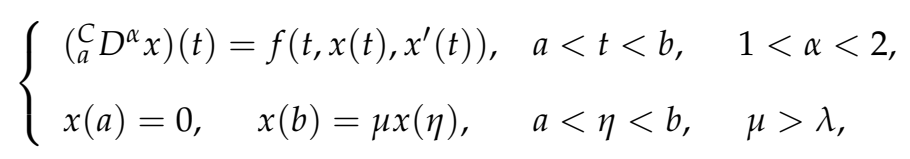

where $\lambda=\frac{b-a}{\eta-a}$ and ${ }_{a}^{C} D^{\alpha}$ stands for the Caputo's fractional derivative, and $f:[a, b] \times \mathbb{R} \times \mathbb{R} \rightarrow \mathbb{R}$ is a continuous function which will be specified later on.

The organization of this paper is as follows. In Section 2, we recall some auxiliary facts and preliminaries. In Section 3, we first find the Green's function associated with (1), and then, using the inequalities related with this function and two well-known fixed-point theorems, we present our main results. An illustrative example is also given.

\section{Preliminaries}

This section is devoted to recall and gathering of some essential definitions and auxiliary facts in fractional calculus, as well as the results needed further on, which can be found in [23-25].

Definition 1. Let $\alpha \geq 0$ and $f$ be a real function defined in $[a, b]$. The Riemann-Liouville fractional integral of order $\alpha$ for a continuous function $f:(a, \infty) \rightarrow \mathbb{R}$ is defined by $\left({ }_{a} I^{0} f\right)(t)=f(t)$ and

$$
\left({ }_{a} I^{\alpha} f\right)(t)=\frac{1}{\Gamma(\alpha)} \int_{a}^{t}(t-s)^{\alpha-1} f(s) d s, \quad \alpha>0, \quad t \in[a, b],
$$

where $\Gamma(\cdot)$ is the Gamma function.

Definition 2. For a continuous function $f:(a, \infty) \rightarrow \mathbb{R}$, the Riemann-Liouville fractional derivative of fractional order $\alpha>0$ is defined by

$$
{ }^{R} D_{a+}^{\alpha} f(t)=\frac{1}{\Gamma(n-\alpha)}\left(\frac{d}{d t}\right)^{n} \int_{a}^{t}(t-s)^{n-\alpha-1} f(s) d s, n=[\alpha]+1,
$$

where $[\alpha]$ denotes the integer part of the real number $\alpha$.

For $\alpha<0$ and the convenience of the reader, we use the denotation $D^{\alpha} y=I^{-\alpha} y$. Moreover, for $\beta \in[0, \alpha)$, it is valid that $D^{\beta} I^{\alpha} y=I^{\alpha-\beta} y$. 
Definition 3. Caputo's fractional derivative of order $\alpha \geq 0$ is given by $\left({ }_{a}^{C} D^{0} f\right)(t)=f(t)$, and $\left({ }_{a}^{C} D^{\alpha} f\right)(t)=$ $\left({ }_{a} I^{m-\alpha} D^{m} f\right)(t)$ for $\alpha>0$, where $m$ is the smallest integer greater or equal to $\alpha$. Besides, it can be formulated by

$$
{ }^{C} D_{a+}^{\alpha} f(t)=\frac{1}{\Gamma(n-\alpha)} \int_{a}^{t}(t-s)^{n-\alpha-1} f^{(n)}(s) d s, n=[\alpha]+1, \quad \text { for } f \in A C^{n}([a, b]),
$$

where $\alpha \notin \mathbb{N}_{0}$ and $A C^{n}([a, b])$ represents the space of all absolutely continuous functions having an absolutely continuous derivative up to $(n-1)$ (see also [23]).

In the sequel, the associated Green's function for the three-point BVP (1) is formulated by utilizing a crucial lemma derived by Zhang [26] as follows:

Lemma 1. Let $\alpha>0$; then, in $C(0, T) \cap L(0, T)$, the differential equation

$$
{ }^{C} D_{0+}^{\alpha} u(t)=0
$$

has solutions $u(t)=c_{0}+c_{1} t+c_{2} t^{2}+\cdots+c_{n} t^{n-1}, c_{i} \in \mathbb{R}, i=0,1, \cdots, n, n=[\alpha]+1$.

Furthermore, it has been proved that $I_{0+}^{\alpha} D_{0+}^{\alpha} u(t)=u(t)+c_{0}+c_{1} t+c_{2} t^{2}+\cdots+c_{n} t^{n-1}$ for some $c_{i} \in \mathbb{R}, i=0,1, \cdots, n, n=[\alpha]+1$ (see Lemma 2.3 in [26]).

\section{Main Results}

In the following, we present a pivotal lemma which will play a crucial role in our next analysis and direct our attention to a variant of Problem (1).

Lemma 2. Let $\Delta:=\mu(\eta-a)-(b-a)>0$. Then, $x \in C^{1}(I, \mathbb{R})$ is the solution of fractional three-point $B V P(1)$ if, and only if $x$ satisfies the integral equation

$$
x(t)=\int_{a}^{b} G(t, s) f\left(s, x(s), x^{\prime}(s)\right) d s, \quad t \in I:=[a, b]
$$

where the Green's function $G(t, s):=G_{1}(t, s)+G_{2}(t, s)$ is given by

$$
\begin{gathered}
G_{1}(t, s)= \begin{cases}\frac{(t-s)^{\alpha-1}}{\Gamma(\alpha)}, & a \leq s \leq t \leq b \\
0, & a \leq t \leq s \leq b\end{cases} \\
G_{2}(t, s)= \begin{cases}\frac{t-a}{\Delta \Gamma(\alpha)}\left((b-s)^{\alpha-1}-\mu(\eta-s)^{\alpha-1}\right), & a \leq s \leq \eta, \quad t \in I, \\
\frac{(b-s)^{\alpha-1}(t-a)}{\Delta \Gamma(\alpha)}, & \eta \leq s \leq b, \quad t \in I .\end{cases}
\end{gathered}
$$

Moreover,

$$
\max _{t, s \in I} G(t, s) \leq \frac{\mu(b-a)^{\alpha}}{\lambda \Delta \Gamma(\alpha)}
$$

Proof. By employing the Riemann-Liouville fractional integral ${ }_{a} I^{\alpha}$ for Equation (1), the imposed boundary conditions, and the knowledge received from the fractional calculus theory, we observe that $x \in C^{1}[a, b]$ is a solution of (1) if, and only if

$$
x(t)=c_{0}+c_{1}(t-a)+\frac{1}{\Gamma(\alpha)} \int_{a}^{t}(t-s)^{\alpha-1} f\left(s, x(s), x^{\prime}(s)\right) d s
$$


for some real constants $c_{0}$ and $c_{1}$ (see Lemma 1 ). Since $x(a)=0$, we immediately derive $c_{0}=0$. Now,

$$
\begin{aligned}
x(b)=\mu x(\eta) \Leftrightarrow & c_{1}(b-a)+\frac{1}{\Gamma(\alpha)} \int_{a}^{b}(b-s)^{\alpha-1} f\left(s, x(s), x^{\prime}(s)\right) d s \\
= & c_{1} \mu(\eta-a)+\frac{\mu}{\Gamma(\alpha)} \int_{a}^{\eta}(\eta-s)^{\alpha-1} f\left(s, x(s), x^{\prime}(s)\right) d s \\
\Leftrightarrow & c_{1}=\frac{1}{\Delta \Gamma(\alpha)}\left(\int_{a}^{b}(b-s)^{\alpha-1} f\left(s, x(s), x^{\prime}(s)\right) d s\right. \\
& \left.-\mu \int_{a}^{\eta}(\eta-s)^{\alpha-1} f\left(s, x(s), x^{\prime}(s)\right) d s\right)
\end{aligned}
$$

which, together with (6), implies that

$$
\begin{aligned}
x(t)= & \frac{1}{\Gamma(\alpha)} \int_{a}^{t}(t-s)^{\alpha-1} f\left(s, x(s), x^{\prime}(s)\right) d s \\
& +\frac{t-a}{\Delta \Gamma(\alpha)}\left(\int_{a}^{b}(b-s)^{\alpha-1} f\left(s, x(s), x^{\prime}(s)\right) d s-\mu \int_{a}^{\eta}(\eta-s)^{\alpha-1} f\left(s, x(s), x^{\prime}(s)\right) d s\right) .
\end{aligned}
$$

This is also equivalent to

$$
\begin{aligned}
x(t)= & \frac{1}{\Gamma(\alpha)} \int_{a}^{t}(t-s)^{\alpha-1} f\left(s, x(s), x^{\prime}(s)\right) d s \\
& +\frac{t-a}{\Delta \Gamma(\alpha)}\left(\int_{a}^{\eta}\left((b-s)^{\alpha-1}-\mu(\eta-s)^{\alpha-1}\right) f\left(s, x(s), x^{\prime}(s)\right) d s\right. \\
& \left.+\int_{\eta}^{b}(b-s)^{\alpha-1} f\left(s, x(s), x^{\prime}(s)\right) d s\right) .
\end{aligned}
$$

Now, (8) can be rewritten as follows:

$$
x(t)=\int_{a}^{b} G(t, s) f\left(s, x(s), x^{\prime}(s)\right) d s, \quad t \in I=[a, b],
$$

where the associated Green's function $G(t, s)=G_{1}(t, s)+G_{2}(t, s)$ is defined by (3) and (4). Furthermore, for any $s \in I$,

$$
\begin{aligned}
\max _{t \in I} G(t, s) & =\frac{(b-s)^{\alpha-1}}{\Gamma(\alpha)}+\max _{t \in I}\left(\frac{(b-s)^{\alpha-1}(t-a)}{\Delta \cdot \Gamma(\alpha)}\right) \\
& =\frac{(b-s)^{\alpha-1}}{\Gamma(\alpha)}\left(1+\frac{b-a}{\Delta}\right) \\
& =\frac{(b-s)^{\alpha-1}}{\Delta \Gamma(\alpha)}(\mu(\eta-a)) \\
& \leq \frac{\mu(b-a)^{\alpha}}{\lambda \Delta \Gamma(\alpha)}
\end{aligned}
$$

Therefore, the inequality (5) is proved.

Throughout the remainder of this paper, we employ two well-known fixed-point results to study Equation (1).

\subsection{Existence of Positive Solution with the Schauder Fixed-Point Principle}

In the following, we investigate Equation (1) via the Schauder fixed-point theorem. 
Theorem 1 (Schauder fixed-point Theorem, [27]). Let $U$ be a nonempty and convex subset of a normed space $X$. Let $T$ be a continuous mapping of $U$ into a compact set $K \subset U$. Then, $T$ has a fixed point.

In the sequel, we suppose the following condition:

$\left(C_{0}\right) f$ satisfies Carathéodory-type conditions. That is, $f(\cdot, u, v)$ is measurable for the fixed $u, v$, and $f(t, \cdot, \cdot)$ is continuous for a.e. $t \in I$. Moreover, if $u \geq 0$, then $f(t, u, v) \geq 0$.

Under this condition, the equivalent representation for Equation (2) is given by

$$
x(t)=[\mathcal{F} x](t), \quad t \in I=[a, b],
$$

where $\mathcal{F}$ is an operator defined by

$$
[\mathcal{F} x](t)=\int_{a}^{b} G(t, s) f\left(s, x(s), x^{\prime}(s)\right) d s, \quad t \in I .
$$

It is obvious to see that $x(t)$ is a solution to the problem (1) if it is a fixed point of the operator $\mathcal{F}$.

Theorem 2. Suppose that $f$ satisfies the condition $\left(C_{0}\right)$ and the followings:

$\left(C_{1}\right)$ There exists an $L^{1}$-function $\varphi: I \rightarrow \mathbb{R}^{+}$, such that

$$
\left|f\left(t, x(t), x^{\prime}(t)\right)\right| \leq \varphi(t) \Omega(\|x\|), \quad x \in C^{1}(I, \mathbb{R}), \quad t \in I,
$$

where $\Omega: I \rightarrow[0, \infty)$ is a non-decreasing continuous function and $\|\cdot\|$ denotes the supremum norm on $I$.

$\left(C_{2}\right)$ The point $\eta \in(a, b)$ is taken sufficiently close to $a$, such that

$$
\mu \int_{a}^{\eta}(\eta-s)^{\alpha-1} f\left(s, x(s), x^{\prime}(s)\right) d s \leq \int_{a}^{b}(b-s)^{\alpha-1} f\left(s, x(s), x^{\prime}(s)\right) d s, \quad \text { for all } x \in C^{1}(I, \mathbb{R}) .
$$

Moreover, suppose that there exists a continuous function $p$ defined on I satisfying the following inequality:

$$
\frac{\mu(b-a)^{\alpha}}{\lambda \Delta \Gamma(\alpha)}\|\varphi\|_{1} \Omega(\|p\|) \leq\|p\| .
$$

Then, Equation (1) has at least one positive solution in $C^{1}(I, \mathbb{R})$, bounded above by $\|p\|$.

Proof. Let us define

$$
S=\left\{x \in C^{1}(I, \mathbb{R}) \mid 0 \leq x(t) \leq\|p\| \text { for } t \in I\right\}
$$

where $\|\cdot\|_{1}$ denotes the $L^{1}$-norm on $I$, and $p$ is a function satisfying the condition $\left(C_{2}\right)$. Clearly, the set $S$ is a non-empty, closed, bounded, and convex subset of $C^{1}(I, \mathbb{R})$. To establish that Equation (1) has a positive solution, it only suffices to show that the operator $\mathcal{F}$ has a fixed point in $S$. We first show that $S$ is $\mathcal{F}$-invariant. Let $x(t)$ be a non-negative function; then, following condition $\left(C_{0}\right)$, one finds that $f\left(t, x(t), x^{\prime}(t)\right)$ is non-negative too, and the right-hand side of $(7)$, together with conditions $\left(C_{0}\right),\left(C_{2}\right)$ and the fact that $\Delta>0$ imply that $[\mathcal{F} x](t) \geq 0$.

On the other hand, using (2), (9), (10) and condition $\left(C_{1}\right)$, one can see that

$$
|[\mathcal{F} x](t)| \leq\|p\|, \quad \text { for } t \in I .
$$


Hence, $\mathcal{F} S \subset S$. Furthermore, to show the continuity of the operator $\mathcal{F}: S \rightarrow S$, we have

$$
\left|\left[\mathcal{F} x_{n}\right](t)-[\mathcal{F} x](t)\right| \rightarrow 0 \Longleftrightarrow \int_{a}^{b}|G(t, s)| \cdot\left|f\left(s, x(s), x^{\prime}(s)\right)-f\left(s, x_{n}(s), x_{n}^{\prime}(s)\right)\right| d s \rightarrow 0
$$

for $x, x_{n} \in S \subset C^{1}(I, \mathbb{R})$.

Next, we show that $\mathcal{F} S$ is equicontinuous. Assume that $a \leq t_{1}<t_{2} \leq b$. Following the definition of $\mathcal{F}$ and the condition $\left(C_{1}\right)$, we have

$$
\left|[\mathcal{F} x]\left(t_{2}\right)-[\mathcal{F} x]\left(t_{1}\right)\right| \leq\|\varphi\|_{1} \Omega(\|x\|) \cdot \max _{s \in I}\left|G\left(t_{2}, s\right)-G\left(t_{1}, s\right)\right|,
$$

which tends to zero, as $t_{1} \rightarrow t_{2}$. Consequently, we conclude that $\mathcal{F} S$ is equicontinuous. Furthermore, the equicontinuity of the set of functions $[\mathcal{F} S]^{\prime}=\left\{y^{\prime}: y=\mathcal{F} x, x \in S\right\}$ can also be shown. Indeed, suppose that $a \leq t_{1}<t_{2} \leq b$; then,

$$
\left|[\mathcal{F} x]^{\prime}\left(t_{2}\right)-[\mathcal{F} x]^{\prime}\left(t_{1}\right)\right| \leq\|\varphi\|_{1} \Omega(\|x\|) \cdot \max _{s \in I}\left|\frac{\partial}{\partial t} G\left(t_{2}, s\right)-\frac{\partial}{\partial t} G\left(t_{1}, s\right)\right| \rightarrow 0
$$

whenever $t_{1} \rightarrow t_{2}$. Therefore, we conclude that $[\mathcal{F} S]^{\prime}$ is equicontinuous.

Besides, $S$ is totally bounded (since every sequence in $S$ has a Cauchy subsequence), so $S$ is compact and $\mathcal{F} S$ is compact. Now, all the conditions of the Schauder fixed point are fulfilled; thus, the operator $\mathcal{F}$ as a self-map on $S$ possesses a fixed point in this set, which yields that Equation (1) has a positive solution bounded above by $\|p\|$.

\subsection{Existence of Positive Solution via the Krasnoselskii Type Fixed-Point Theorem}

In what follows, we recall a generalization of Krasnoselskii's fixed-point theorem of cone expansion and compression of a norm type. To do this, let us suppose $(X,\|\cdot\|)$ is a Banach space, and $P$ is the cone in $X$. Assume that $\bar{\alpha}, \bar{\beta}: X \rightarrow \mathbb{R}^{+}$are two continuous non-negative functionals that satisfy

$$
\bar{\alpha}(r x) \leq|r| \bar{\alpha}(x), \quad \bar{\beta}(r x) \leq|r| \bar{\beta}(x), \quad \text { for } x \in X, r \in[0,1],
$$

and

$$
M_{1} \max \{\bar{\alpha}(x), \bar{\beta}(x)\} \leq\|x\| \leq M_{2} \max \{\bar{\alpha}(x), \bar{\beta}(x)\}, \quad \text { for } x \in X,
$$

where $M_{1}, M_{2}$ are two positive constants.

The following lemma is understood as a special case of a result derived by Bai and Ge (see [22] Theorem 2.1).

Lemma 3. Let $r_{2}>r_{1}>0, L_{2}>L_{1}>0$ be constants and

$$
\Omega_{i}=\left\{x \in X \mid \bar{\alpha}(x)<r_{i}, \bar{\beta}(x)<L_{i}\right\}, i=1,2
$$

be two open subsets in $X$, such that $\theta \in \Omega_{1} \subset \bar{\Omega}_{1} \subset \Omega_{2}$. In addition, let

$$
\begin{aligned}
& C_{i}=\left\{x \in X \mid \bar{\alpha}(x)=r_{i}, \bar{\beta}(x) \leq L_{i}\right\}, i=1,2 \\
& D_{i}=\left\{x \in X \mid \bar{\alpha}(x) \leq r_{i}, \bar{\beta}(x)=L_{i}\right\}, i=1,2 .
\end{aligned}
$$

Assume $T: P \rightarrow P$ is a completely continuous operator satisfying

$\left(S_{1}\right) \bar{\alpha}(T x) \leq r_{1}, x \in C_{1} \cap P ; \bar{\beta}(T x) \leq L_{1}, x \in D_{1} \cap P ;$

$\bar{\alpha}(T x) \geq r_{2}, x \in C_{2} \cap P ; \bar{\beta}(T x) \geq L_{2}, x \in D_{2} \cap P ;$ 
or

(S 2$) \bar{\alpha}(T x) \geq r_{1}, x \in C_{1} \cap P ; \bar{\beta}(T x) \geq L_{1}, x \in D_{1} \cap P$;

$\bar{\alpha}(T x) \leq r_{2}, x \in C_{2} \cap P ; \bar{\beta}(T x) \leq L_{2}, x \in D_{2} \cap P ;$

then, $T$ has at least one fixed point in $\left(\bar{\Omega}_{2} \backslash \Omega_{1}\right) \cap P$.

To apply the recent fixed-point theorem, let us consider the following settings.

Let $X$ be a Banach space in $C^{1}(I, \mathbb{R})$, with

$$
\|x\|=\max \left\{\max _{t \in I}|x(t)|, \max _{t \in I}\left|x^{\prime}(t)\right|\right\}, \quad x \in X .
$$

Define a cone $P$ by

$$
P=\{x \in X \mid x(t) \geq 0, \quad \text { for all } t \in I\},
$$

and functionals

$$
\bar{\alpha}(x)=\max _{t \in I}|x(t)|, \quad \bar{\beta}(x)=\max _{t \in I}\left|x^{\prime}(t)\right|, \quad \forall x \in X .
$$

With the help of (11) and (12), $\bar{\alpha}$ and $\bar{\beta}$ are two continuous non-negative functionals, such that $\|x\|=\max \{\bar{\alpha}(x), \bar{\beta}(x)\}$. Let us consider the following notations:

$$
\begin{gathered}
L:=\max _{t \in I}\left|\int_{a}^{b} G(t, s) d s\right| \\
N:=\left(\frac{(b-a)^{\alpha-1}}{\Gamma(\alpha)}+\frac{1}{\Delta \Gamma(\alpha+1)}\left[2(b-\xi)^{\alpha}-2 \mu(\eta-\xi)^{\alpha}+\mu(\eta-a)^{\alpha}-(b-a)^{\alpha}\right]\right),
\end{gathered}
$$

where $\xi=\eta-\frac{b-\eta}{\mu^{\frac{1}{\alpha-1}}-1} \in[a, \eta]$.

Accounting on condition $\left(C_{2}\right)$, we get that the operator $\mathcal{F}$ (as defined before) transforms $P$ into itself; moreover, a standard argument shows that it is completely continuous. In fact, $\mathcal{F}$ is continuous and maps any bounded subset of $P$ into a relatively compact subset of $P$.

In the following result, we suppose that $\eta$ is sufficiently close to $a$ such that the Green function $G$ is non-negative. For the possibility, we refer to Example (1).

Theorem 3. Suppose there are four constants, $k_{2}>k_{1}>0, l_{2}>l_{1}>0$, such that $\max \left\{\frac{k_{1}}{L}, \frac{l_{1}}{M}\right\} \leq$ $\min \left\{\frac{k_{2}}{L}, \frac{l_{2}}{N}\right\}$, and the following assumptions hold:

$\left(C_{3}\right)$ There is an $L^{1}$-function $\psi: I \rightarrow \mathbb{R}^{+}$which satisfies the following condition:

$$
\int_{a}^{b}(b-s)^{\alpha-1} f\left(s, x(s), x^{\prime}(s)\right) d s-\mu \int_{a}^{\eta}(\eta-s)^{\alpha-1} f\left(s, x(s), x^{\prime}(s)\right) d s \geq \int_{a}^{b} \psi(s) f\left(s, x(s), x^{\prime}(s)\right) d s,
$$

for all $x \in C^{1}\left(I, \mathbb{R}^{+}\right)$.

(C. $f(t, u, v) \geq \max \left\{\frac{k_{1}}{L}, \frac{l_{1}}{M}\right\}$, for $(t, u, v) \in I \times\left[0, k_{1}\right] \times\left[-l_{1}, l_{1}\right]$;

$\left(C_{5}\right) f(t, u, v) \leq \min \left\{\frac{k_{2}}{L}, \frac{l_{2}}{N}\right\}$, for $(t, u, v) \in I \times\left[0, k_{2}\right] \times\left[-l_{2}, l_{2}\right]$, 
where $M=\frac{\|\psi\|_{1}}{\Delta \Gamma(\alpha)}$. Then, problem (1) has at least one positive solution $x(t)$, such that

$$
k_{1} \leq \max _{t \in I} x(t) \leq k_{2} \quad \text { or } \quad l_{1} \leq \max _{t \in I}\left|x^{\prime}(t)\right| \leq l_{2} .
$$

Proof. Let us take the following subsets of $X=C^{1}(I, \mathbb{R})$

$$
\begin{array}{lll}
\Omega_{i}=\left\{x \in X \mid \bar{\alpha}(x)<k_{i},\right. & \left.\bar{\beta}(x)<l_{i}\right\}, & i=1,2 ; \\
\mathcal{P}_{i}=\left\{x \in X \mid \bar{\alpha}(x)=k_{i},\right. & \left.\bar{\beta}(x) \leq l_{i}\right\}, & i=1,2 ; \\
\mathcal{Q}_{i}=\left\{x \in X \mid \bar{\alpha}(x) \leq k_{i},\right. & \left.\bar{\beta}(x)=l_{i}\right\}, & i=1,2 .
\end{array}
$$

For $x \in \mathcal{P}_{1} \cap P$, by $\left(C_{4}\right)$, there exists

$$
\bar{\alpha}(\mathcal{F} x)=\max _{t \in I}\left|\int_{a}^{b} G(t, s) f\left(s, x(s), x^{\prime}(s)\right) d s\right| \geq \frac{k_{1}}{L} \max _{t \in I}\left|\int_{a}^{b} G(t, s) d s\right|=k_{1} .
$$

Since $\eta$ is taken sufficiently close to $a$ such that the Green function $G$ is non-negative, the inequality (13) holds. Moreover, taking into account the continuity and properties of $\mathcal{F}$, we derive

$$
\begin{aligned}
(\mathcal{F} x)^{\prime}(t)= & \frac{\alpha-1}{\Gamma(\alpha)} \int_{a}^{t}(t-s)^{\alpha-2} f\left(s, x(s), x^{\prime}(s)\right) d s \\
& +\frac{1}{\Delta \Gamma(\alpha)}\left(\int_{a}^{b}(b-s)^{\alpha-1} f\left(s, x(s), x^{\prime}(s)\right) d s-\mu \int_{a}^{\eta}(\eta-s)^{\alpha-1} f\left(s, x(s), x^{\prime}(s)\right) d s\right), \\
(\mathcal{F} x)^{\prime \prime}(t)= & -\frac{(\alpha-1)(2-\alpha)}{\Gamma(\alpha)} \int_{a}^{t}(t-s)^{\alpha-3} f\left(s, x(s), x^{\prime}(s)\right) d s \leq 0, \quad t \in I .
\end{aligned}
$$

Therefore, $(\mathcal{F} x)(t)$ is concave on $I$, and so the absolute value of $(\mathcal{F} x)^{\prime}$ takes its maximum only at the endpoints of $I$. That is,

$$
\max _{t \in I}\left|(\mathcal{F} x)^{\prime}(t)\right|=\max _{t \in I}\left\{\left|(\mathcal{F} x)^{\prime}(a)\right|,\left|(\mathcal{F} x)^{\prime}(b)\right|\right\}=\left|(\mathcal{F} x)^{\prime}(b)\right| .
$$

Therefore, for $x \in \mathcal{Q}_{1} \cap P$, followed by $\left(C_{3}\right)$ and $\left(C_{4}\right)$, one can see that

$$
\begin{aligned}
\beta(\mathcal{F} x) & =\max _{t \in I}\left\{\left|(\mathcal{F} x)^{\prime}(a)\right|,\left|(\mathcal{F} x)^{\prime}(b)\right|\right\} \\
& \geq\left|(\mathcal{F} x)^{\prime}(a)\right| \\
& =\frac{1}{\Delta \Gamma(\alpha)}\left(\int_{a}^{b}(b-s)^{\alpha-1} f\left(s, x(s), x^{\prime}(s)\right) d s-\mu \int_{a}^{\eta}(\eta-s)^{\alpha-1} f\left(s, x(s), x^{\prime}(s)\right) d s\right) \\
& \geq \frac{1}{\Delta \Gamma(\alpha)} \int_{a}^{b} \psi(s) f\left(s, x(s), x^{\prime}(s)\right) d s \\
& \geq \frac{l_{1}}{M \Delta \Gamma(\alpha)}\|\psi\|_{1}=l_{1} .
\end{aligned}
$$

Now, assuming $x \in \mathcal{P}_{2} \cap P$, by $\left(C_{5}\right)$, there is

$$
\begin{aligned}
\bar{\alpha}(\mathcal{F} x) & =\max _{t \in I}\left|\int_{a}^{b} G(t, s) f\left(s, x(s), x^{\prime}(s)\right) d s\right| \\
& \leq \frac{k_{2}}{L} \max _{t \in I}\left|\int_{a}^{b} G(t, s) d s\right|=k_{2} .
\end{aligned}
$$


Finally, for $x \in \mathcal{Q}_{2} \cap P$, by $\left(C_{5}\right)$, one can find

$$
\begin{aligned}
\beta(\mathcal{F} x)= & \max _{t \in I}\left\{\left|(\mathcal{F} x)^{\prime}(a)\right|,\left|(\mathcal{F} x)^{\prime}(b)\right|\right\} \\
= & \left|(\mathcal{F} x)^{\prime}(b)\right| \\
= & \frac{\alpha-1}{\Gamma(\alpha)} \int_{a}^{b}(b-s)^{\alpha-2} f\left(s, x(s), x^{\prime}(s)\right) d s \\
& +\frac{1}{\Delta \Gamma(\alpha)}\left(\int_{a}^{\eta}\left((b-s)^{\alpha-1}-\mu(\eta-s)^{\alpha-1}\right) f\left(s, x(s), x^{\prime}(s)\right) d s\right. \\
& \left.+\int_{\eta}^{b}(b-s)^{\alpha-1} f\left(s, x(s), x^{\prime}(s)\right) d s\right) \\
\leq & \frac{l_{2}}{N}\left(\frac{(b-a)^{\alpha-1}}{\Gamma(\alpha)}+\frac{1}{\Delta \Gamma(\alpha)} \int_{a}^{\eta}\left|(b-s)^{\alpha-1}-\mu(\eta-s)^{\alpha-1}\right| d s+\frac{(b-\eta)^{\alpha}}{\Delta \Gamma(\alpha+1)}\right) \\
= & \frac{l_{2}}{N}\left(\frac{(b-a)^{\alpha-1}}{\Gamma(\alpha)}+\frac{1}{\Delta \Gamma(\alpha)}\left(\int_{a}^{\xi}\left[\mu(\eta-s)^{\alpha-1}-(b-s)^{\alpha-1}\right] d s\right.\right. \\
& \left.\left.+\int_{\xi}^{\eta}\left[(b-s)^{\alpha-1}-\mu(\eta-s)^{\alpha-1}\right] d s\right)+\frac{(b-\eta)^{\alpha}}{\Delta \Gamma(\alpha+1)}\right) \\
= & \frac{l_{2}}{N}\left(\frac{(b-a)^{\alpha-1}}{\Gamma(\alpha)}+\frac{1}{\Delta \Gamma(\alpha+1)}\left[2(b-\xi)^{\alpha}-2 \mu(\eta-\xi)^{\alpha}+\mu(\eta-a)^{\alpha}-(b-a)^{\alpha}\right]\right) \\
= & l_{2} \cdot
\end{aligned}
$$

Now, all conditions of Lemma 3 are satisfied, and it implies that there exists $x \in\left(\bar{\Omega}_{2} \backslash \Omega_{1}\right) \cap P$, such that $x=\mathcal{F} x$. That is, the problem (1) has at least one positive solution $x(t)$, such that

$$
k_{1} \leq \bar{\alpha}(x) \leq k_{2} \quad \text { or } \quad l_{1} \leq \bar{\beta}(x) \leq l_{2}
$$

In other words,

$$
k_{1} \leq \max _{t \in I} x(t) \leq k_{2} \quad \text { or } \quad l_{1} \leq \max _{t \in I}\left|x^{\prime}(t)\right| \leq l_{2}
$$

which completes the proof.

In the following, we illustrate the said result with an example.

Example 1. Consider the boundary value problem:

$$
\left\{\begin{array}{l}
\left({ }_{a}^{C} D^{\frac{3}{2}} x\right)(t)=f\left(t, x(t), x^{\prime}(t)\right), \quad 0<t<1, \\
x(0)=0, \quad x(1)=\mu x(\eta), \quad 0<\eta<1, \quad \mu \eta>1,
\end{array}\right.
$$

where $\mu=a \eta^{-r}$ for some $a>1,0<r<1.5$, and $f:[0,1] \times \mathbb{R} \times \mathbb{R} \rightarrow \mathbb{R}$ is given by

$$
f(t, u, v)=\lambda_{1} \sin ^{2} u+\lambda_{2} \cos ^{2} v+\lambda_{3} t+\lambda_{4}, \quad t \in I=[0,1], u, v \in \mathbb{R}
$$

such that $\lambda_{i} \geq 0, \lambda_{4}>0, i=1,2,3$, are constant. Since $f$ takes both supremum and infimum over domain $D$, let us set

$$
\inf _{D} f(t, u, v)=M_{1}, \quad \sup _{D} f(t, u, v)=M_{2}
$$

A direct computation shows that

$$
L=\frac{4 \mu \lambda}{3(\mu \lambda-1) \sqrt{\pi}} .
$$


On the other hand, by considering $\eta$ as being sufficiently close to 0 and $\psi(t)<\frac{2 M_{1}}{3 M_{2}}$, we see that

$$
\int_{0}^{1} \sqrt{1-s} f\left(s, x(s), x^{\prime}(s)\right) d s>\int_{0}^{1} \psi(s) f\left(s, x(s), x^{\prime}(s)\right) d s .
$$

Thus, condition $\left(C_{3}\right)$ is satisfied. To give more detail, if $\eta \rightarrow 0$, then using the fact that $\mu=a \eta^{-r}$, together with Leibniz's rule, we see that

$$
\begin{aligned}
\lim _{\eta \rightarrow 0}\left|\mu \int_{0}^{\eta} \sqrt{\eta-s} f\left(s, x(s), x^{\prime}(s)\right) d s\right| & =\frac{a}{2 r} \lim _{\eta \rightarrow 0} \frac{\left|\int_{0}^{\eta} \frac{1}{\sqrt{\eta-s}} f\left(s, x(s), x^{\prime}(s)\right) d s\right|}{\eta^{r-1}} \\
& \leq \frac{a\left|M_{2}\right|}{2 r} \lim _{\eta \rightarrow 0} \frac{\int_{0}^{\eta} \frac{1}{\sqrt{\eta-s}} d s}{\eta^{r-1}} \\
& =\frac{a\left|M_{2}\right|}{r} \lim _{\eta \rightarrow 0} \eta^{1.5-r}=0,
\end{aligned}
$$

which shows that the second integral term in the left-hand side of the inequality in condition $\left(C_{3}\right)$ vanishes for $\eta$ sufficiently close to 0.

$$
\text { Furthermore, } \begin{aligned}
M & =\frac{2\|\psi\|_{1}}{(\mu \eta-1) \sqrt{\pi}}<L \text { and } \\
N & =\frac{2}{\sqrt{\pi}}+\frac{L}{\mu \eta}\left(2(1-\xi)^{1.5}-2 \mu(\eta-\xi)^{1.5}+\mu \eta^{1.5}-1\right)>L,
\end{aligned}
$$

where $\xi=\frac{\eta \mu^{2}-1}{\mu^{2}-1} \in(0,1)$. Next, to check the conditions $\left(C_{4}\right)$ and $\left(C_{5}\right)$, choose $k_{2}>l_{2}>l_{1}>k_{1}>0$, such that $l_{1}=M \cdot M_{1}$ and $l_{2}=N \cdot M_{2}$. Then, one can derive the followings:

$$
\begin{aligned}
& f(t, u, v) \geq \max \left\{\frac{k_{1}}{L}, \frac{l_{1}}{M}\right\}=\frac{l_{1}}{M}, \quad \text { for }(t, u, v) \in I \times\left[0, k_{1}\right] \times\left[-l_{1}, l_{1}\right] ; \\
& f(t, u, v) \leq \min \left\{\frac{k_{2}}{L}, \frac{l_{2}}{N}\right\}=\frac{l_{2}}{N}, \quad \text { for }(t, u, v) \in I \times\left[0, k_{2}\right] \times\left[-l_{2}, l_{2}\right] .
\end{aligned}
$$

That is to say, all the assumptions of Theorem 3 are fulfilled, then problem (14) has at least one positive solution $x$, such that

$$
k_{1} \leq \max _{t \in I} x(t) \leq k_{2} \quad \text { or } \quad M \cdot M_{1} \leq \max _{t \in I}\left|x^{\prime}(t)\right| \leq N \cdot M_{2} .
$$

Author Contributions: Formal Analysis, E.P., R.S. and S.K.N.

Acknowledgments: The authors thank the reviewers for their useful remarks on our work.

Conflicts of Interest: The authors declare no conflict of interest.

\section{References}

1. Gupta, C.P. Solvability of a three-point nonlinear boundary value problem for a second order ordinary differential equation. J. Math. Anal. Appl. 1992, 168, 540-551. [CrossRef]

2. Ma, R. Multiplicity of positive solutions for second-order three-point boundary value problems. Comput. Math. Appl. 2000, 40, 193-204. [CrossRef]

3. He, X.; Ge, W. Triple solutions for second-order three-point boundary value problems. J. Math. Anal. Appl. 2002, 268, 256-265. [CrossRef] 
4. Leggett, R.W.; Williams, L.R. Multiple positive fixed-points of nonlinear operators on ordered Banach spaces. Indiana Univ. Math. J. 1979, 28, 673-688. [CrossRef]

5. Bai, Z. Solvability for a class of fractional $m$-point boundary value problem at resonance. Comput. Math. Appl. 2011, 62, 1292-1302. [CrossRef]

6. Bai, Z.; Zhang, Y. Solvability of fractional three-point boundary value problems with nonlinear growth. Appl. Math. Comput. 2011, 218, 1719-1725. [CrossRef]

7. Cui, Y.; Sun, J. Positive solutions for second-order three-point boundary value problems in Banach spaces. Acta Math. Sin. 2011, 4, 743-751.

8. Guo, Y.; Ge, W. Positive solutions for three-point boundary value problems with dependence on the first order derivative. J. Math. Anal. Appl. 2004, 290, 291-301. [CrossRef]

9. Il'in, V.A.; Moiseev, E.I. Nonlocal boundary value problem of the first kind for a Sturm-Liouville operator in its differential and finite difference aspects. Differ. Equ. 1987, 23, 803-810.

10. Il'in, V.A.; Moiseev, E.I. Nonlocal boundary value problem of the second kind for a Sturm-Liouville operator. Differ. Equ. 1987, 23, 979-987.

11. Ji, D.; Bai, Z.; Ge, W. The existence of countably many positive solutions for singular multipoint boundary value problems. Nonlinear Anal. Theory Methods Appl. 2010, 72, 955-964. [CrossRef]

12. Li, H. Existence of nontrivial solutions for superlinear three-point boundary value problems. Acta Math. Appl. Sin. 2017, 33, 1043-1052. [CrossRef]

13. Ma, R. Positive solutions of nonlinear three-point boundary value problem. Electron. J. Differ. Equations 1999, 34, 1-8. [CrossRef]

14. Marano, S. A remark on a second order three-point boundary value problem. J. Math. Anal. Appl. 1994, 183, 518-522. [CrossRef]

15. Webb, J.R.L. Positive solutions of some three point boundary value problems via fixed-point index theory. Nonlinear Anal. Theory Methods Appl. 2001, 47, 4319-4332. [CrossRef]

16. Ntouyas, S.K.; Pourhadi, E. Positive solutions of nonlinear fractional three-point boundary-value problem. Le Mat. 2018, 73, 139-154.

17. Sudsutad, W.; Tariboon, J.; Ntouyas, S.K. Positive solutions for fractional differential equations with three-point multi-term fractional integral boundary conditions. Adv. Differ. Equ. 2014, 2014, 28.

18. Wang, G.; Ntouyas, S.K.; Zhang, L. Positive solutions of the three-point boundary value problem for fractional-order differential equations with an advanced argument. Adv. Differ. Equ. 2011, 2011, 2. [CrossRef]

19. Wang, G.; Zhang, L.; Ntouyas, S.K. Multiplicity of positive solutions for fractional order three-point boundary value problems. Commun. Appl. Nonlinear Anal. 2013, 20, 41-53.

20. Zhang, Y. Existence results for a coupled system of nonlinear fractional multi-point boundary value problems at resonance. J. Inequal. Appl. 2018, 2018, 198. [CrossRef]

21. Zou, Y.; Liu, L.; Cui, Y. The existence of solutions for four-point coupled boundary value problems of fractional differential equations at resonance. Abstr. Appl. Anal. 2014, 2014, 1-8. [CrossRef]

22. Bai, Z.; Ge, W. Existence of positive solutions to fourth-order quasilinear boundary value problems. Acta Math. Sin. 2006, 22, 1825-1830. [CrossRef]

23. Kilbas, A.A.; Srivastava, H.M.; Trujillo, J.J. Theory and Applications of Fractional Differential Equations; North-Holland Math. Stud.; Elsevier: Amsterdam, The Netherlands, 2006; Volume 204.

24. Podlubny, I. Fractional Differential Equations; Mathematics in Science and Engineering; Academic Press: New York, NY, USA; London, UK; Toronto, ON, Canada, 1999; Volume 198.

25. Samko, S.G.; Kilbas, A.A.; Marichev, O.I. Fractional Integral and Derivatives (Theory and Applications); Gordon and Breach: Yverdon, Switzerland, 1993.

26. Zhang, S. Positive solutions for boundary-value problems of nonlinear fractional differential equations. Electron. J. Differ. Equ. 2006, 2006, 1-12. [CrossRef]

27. Smart, D.R. Fixed-Point Theorems; Cambridge University Press: London, UK; New York, NY, USA, 1974.

(C) 2019 by the authors. Licensee MDPI, Basel, Switzerland. This article is an open access article distributed under the terms and conditions of the Creative Commons Attribution (CC BY) license (http:/ / creativecommons.org/licenses/by/4.0/). 